\title{
Multi-Resonating UWB Printed Monopole
}

\section{Antenna}

\author{
Sanjay Singh Thakur, ZaidPanhalkar, AditiSathe
}

\begin{abstract}
The multi-resonating, Annular Ring with Diamond Patch UWB Antenna has been presented, that produces large bandwidth. This configuration shows the bandwidth for VSWR = 2, or for corresponding $S_{11}$ of $1.4 \mathrm{GHz}-11 \mathrm{GHz}$, which includes UWB. This proposed configuration shows, approximately, Omni-directional radiation pattern on azimuthal plane for the entire range of frequency band. The measured and simulated results are shown; they promise for agreeable similarity. The impedance bandwidth ratio for presented antenna is achieved better than 7.85: 1 for $S_{11}<-9.6 \mathrm{~dB}$. This antenna combines two resonators, i.e. annular ring and diamond shaped patch, within FR4 substrate of dimension $80 \mathrm{~mm} \times 80 \mathrm{~mm}$. This low profile compact antenna can be very useful for many embedded systems.
\end{abstract}

Index Terms - Dual Polarization, Annular ring patch, Diamond patch, Multi-resonating antenna, Printed Monopole Antenna, Ultra wideband.

\section{INTRODUCTION}

In present day, the applications under wireless communications have been increased. The wireless communication system requires high data rate transfer. To support high data rate, antenna plays vital role, it must be broadband. For implementing broadband antenna either multi-resonating or multi-mode antenna can be used [1-2]. Therefore, requirement for multi-resonating antennas, that is antenna which have larger bandwidth and smaller area, is tremendously increasing. All these are showing broad/ ultra wideband characteristic and they are working on multi-mode principle; whereas multi-resonating can also provide the broadband/ ultra wideband characteristics [3-7].

In the presented paper, a multi-resonating printed monopole antenna(MPMA) is planned, leading to a new configuration. This configuration of MPMA consist of a diamond which is enclosed by a ring and out of the four vertices of the diamond shaped radiator, two vertices are overlapped or merged with the ring and remaining two edges kept open. The proposed antenna has been analyzed for the parameters like, gain, efficiency, impedance BW, and radiation patterns. This MPMA Antenna has numerous applications such as, the presented antenna can cover frequency bandwidth from $1.40 \mathrm{GHz}$ to $11 \mathrm{GHz}$ and beyond, so that all the presently available wireless communication systems can be included such as GPS, GSM1800, PCS1900,

Revised Version Manuscript Received on 16 September, 2019.

Sanjay Singh Thakur, Vidyalankar Institute of Technology, Wadala East, Mumbai, Maharashtra, India.

(Email: sanjaysingh.thakur@vit.edu.in)

ZaidPanhalkar, Vidyalankar Institute of Technology,Wadala East, Mumbai, Maharashtra, India.

(Email: zaidpanhalkar786@gmail.com)

AditiSathe,Vidyalankar Institute of Technology,Wadala East, Mumbai, Maharashtra, India.

(Email: aditiss14@gmail.com)
WCDMA / UMTS (3G), 2.45 /5.2 /5.8-GHz-ISM, U-NII, DECT, WLANs and UWB (3.1-10.6 GHz) [1-8].

\section{DESIGN OF ANTENNA}

The annular ring antenna with a diamond patch within it is shown in Fig. 1. The current vectors are spreading on the edges of the patch [3-4]. From this one can conclude that the performance is not reliant on the central part of the patch. Hence this central part of circular is cut, making it an annular ring. Annular ring resonates at lower frequency and to excite higher order modes at high frequency the diamond shape patch has been inserted within the annular ring which will excite the higher order modes [8]. Circular patch with radius of $\mathrm{R} 1$ is converted into annular ring with the radius of $\mathrm{R} 2$ selected for inner part, R2 decide the lower end frequency resonance. When difference of $(\mathrm{R} 1-\mathrm{R} 2)$ is kept on decreasing then the impedance of feed point will be increasing and it is not easy to equal the 'fifty' Ohm line. A diamond shaped geometry is introduced inside the annular ring, that may deliver good match and provide considerable reduction of the size of the radiating patch at the same time it helps to excite the higher order modes. The presented antenna has 2 resonators, with sufficient bandwidth, to ensure the achievement of ultra wide bandwidth. The presented antenna geometry is developed in the laboratory with low priced glass epoxy (FR4) substrate with the loss tangent, $\tan \delta=0.01$, relative dielectric constant of $\in r=4.3$, and the thickness of the substrate is $\mathrm{h}=1.59 \mathrm{~mm}$.

\section{Diamond Patch within Annular Ring}

Fig. 1 shows the geometry of presented antenna with physical dimension of size of $80 \mathrm{~mm}$ x $80 \mathrm{~mm}$. The dimension of the proposed patch radiator is linked with lower end of frequency bandwidth. For this proposed antenna the outer radius $\mathrm{R} 1=25 \mathrm{~mm}$, inner radius $\mathrm{R} 2=23 \mathrm{~mm}$, which has been optimized with ring width of $2 \mathrm{~mm}$. The diamond shaped patch has been incorporated within annular ring with dimension of, long diagonal is $50 \mathrm{~mm}$ and short diagonal is of $30 \mathrm{~mm}$. The feed, microstrip line, along with the lower edge of annular ring or the radiating antenna and partial ground plane, behaves as quarter wavelength transformer to match the impedance. For appropriate impedance matching between the patch and the microstrip line feed, is provided with feed-width $=3 \mathrm{~mm}$. The dimension of ground plane of $80 \mathrm{~mm}$ $\mathrm{x} 10 \mathrm{~mm}$ as partial ground plane on back side and the feed gap $(=p)$ of microstrip line feed is optimized to be, as 0.5 $\mathrm{mm}$.For maximum power transformation to the radiating 
patch from the microstrip line feed one may provide better coupling among both the radiating patches. Hence the bandwidth is enhanced.

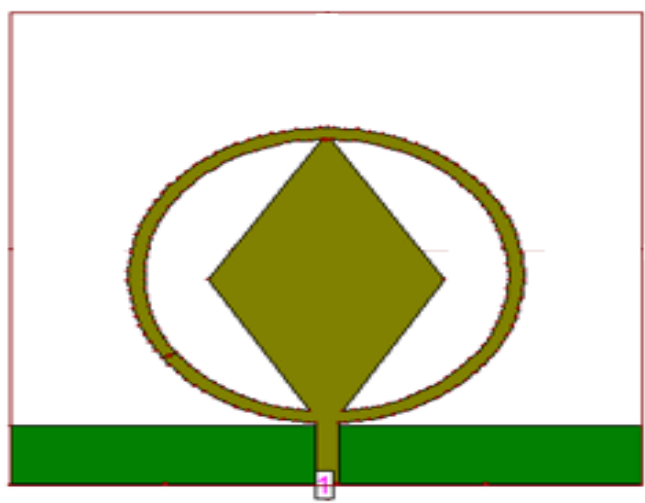

Fig. 1: Annular Ring combind with Diamond Patch

\section{RESULTS AND DISCUSSION}

The lower band edge frequency $\left(\mathrm{f}_{\mathrm{L}}\right)$ for $\mathrm{VSWR}<2$ or $\mathrm{S}_{11}=-9.6 \mathrm{~dB}$ is given by:

$\mathrm{f}_{\mathrm{L}}=\mathrm{c} / \lambda=[7.2 /(1+\mathrm{r}+\mathrm{p}) \mathrm{k}] \mathrm{GHz}$

In given equation 1 , length 1 , which decides lower edge frequency of printed antenna, in which $r$ is the effective radius of an equivalent cylindrical monopole antenna and feed gap is specified by $\mathrm{p}$ and the correction factor $\mathrm{k}$ has been selected as 1.1 for the substrate glass epoxy [4-8]. All parameter $1, \mathrm{r}$ and $\mathrm{p}$ are in $\mathrm{cm}$ which produces $\mathrm{f}$ in $\mathrm{GHz}$. Different parameters of designed antenna have been optimized to achieve impedance bandwidth in the span of 1.4

The characteristics of the presented antenna have been simulated by using IE3D software [9]. Different geometries and corresponding return losses $S_{11}$ are shown in fig. 2 . The optimized configuration is fabricated and the photograph is shown in fig. 3 .

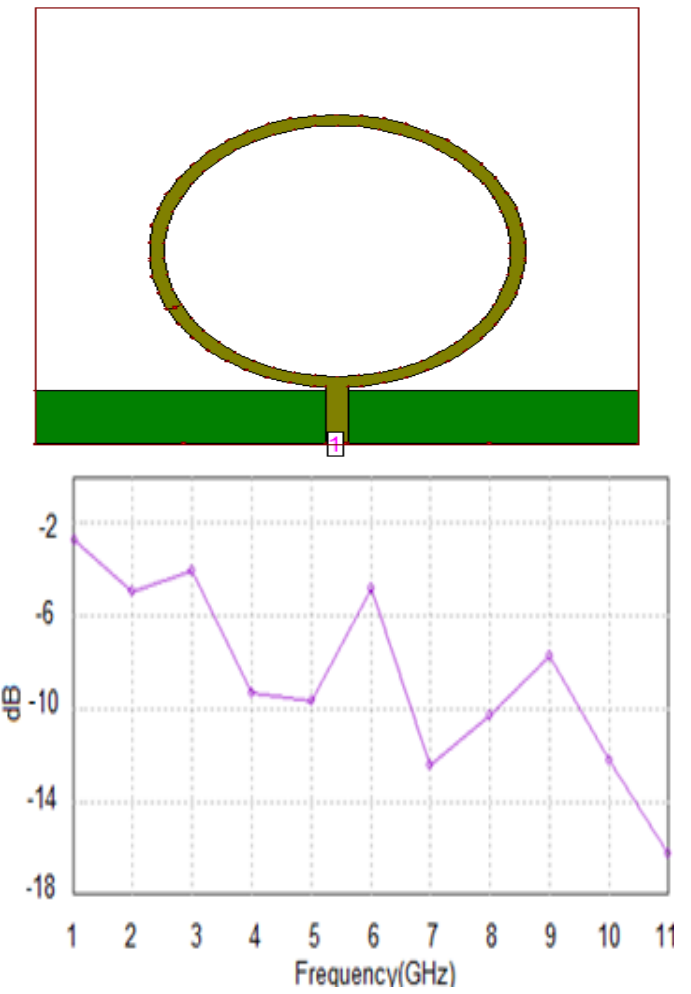

(a) Annular Ring's Return Loss $\mathrm{GHz}$ to $11 \mathrm{GHz}$.
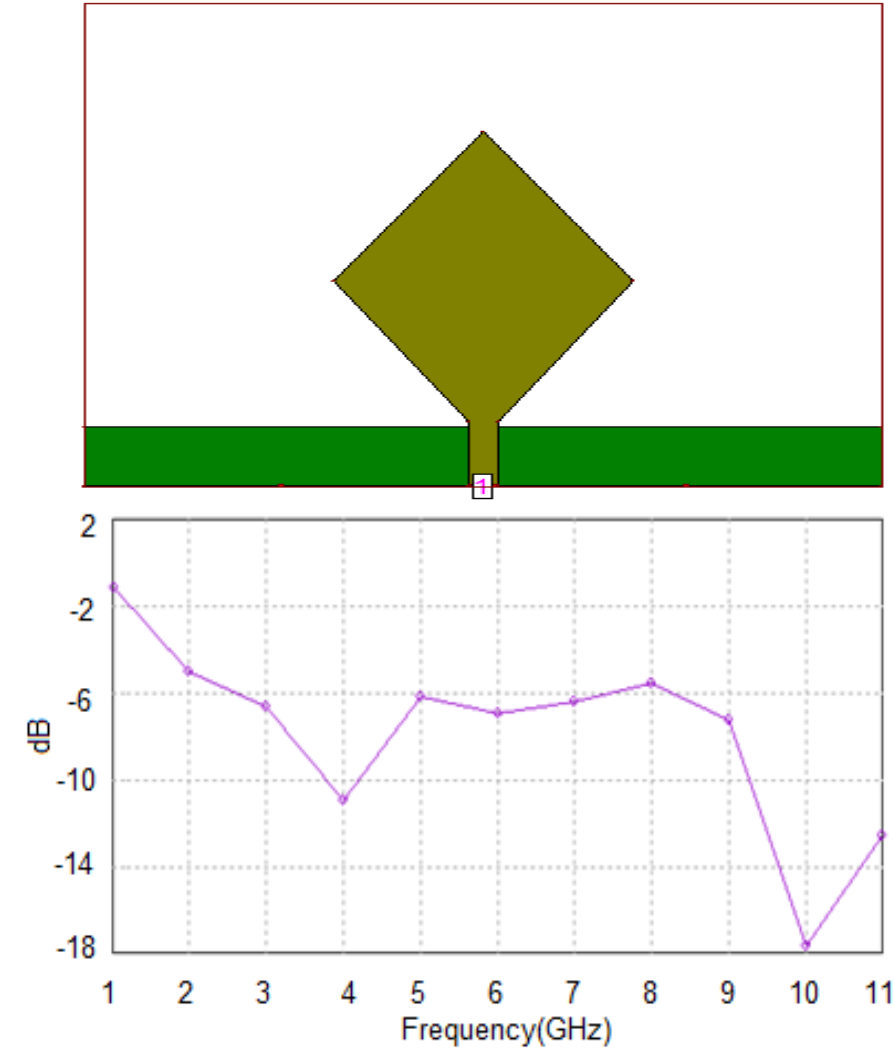

(b) Diamond's Return Loss

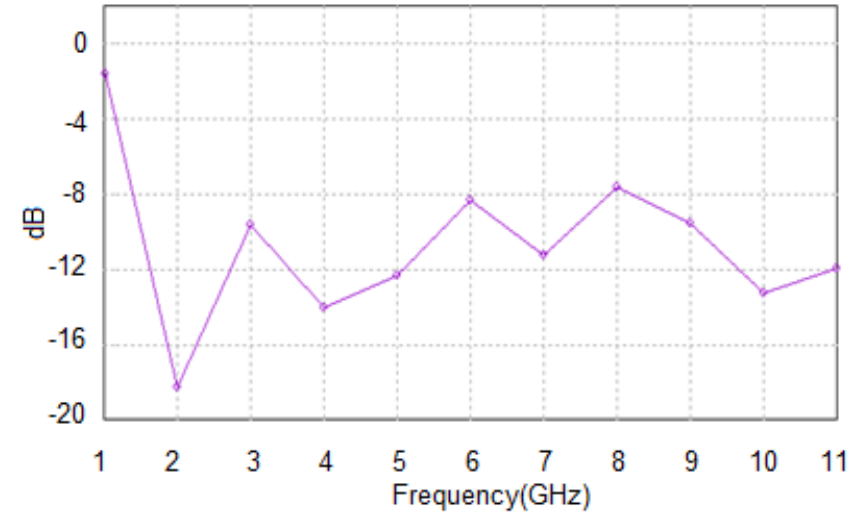

(c) Annular Ring combind with Diamond Patch's (Fig.1) Return Loss

Fig. 2: Different geometries

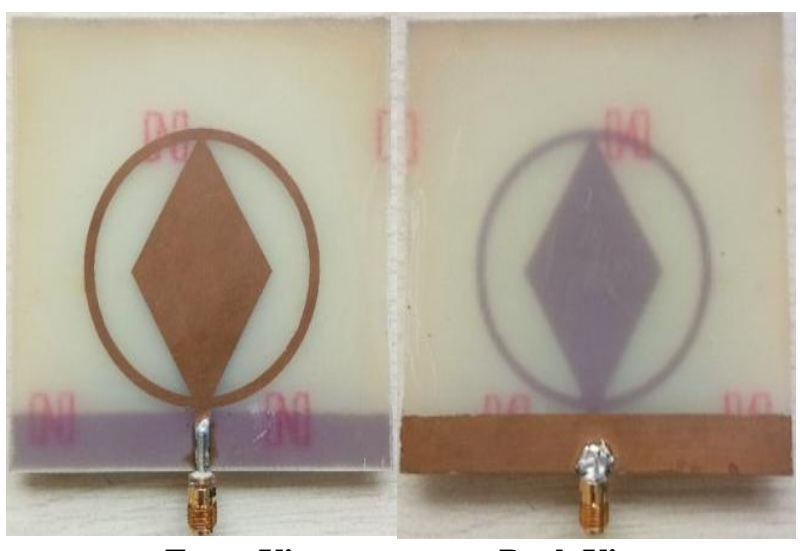

Front View Back View

Fig3: Photograph of prototype

Published By:

Blue Eyes Intelligence Engineering 
The simulated return loss is shown in fig. 4 and measured results are shown to validate the same. The bandwidth has been checked for proposed antenna, found to be over $1.4 \mathrm{GHz}$ to $11 \mathrm{GHz}$. Simulated $\mathrm{S}_{11}$ in $\mathrm{dB}$ wrt to frequency in $\mathrm{GHz}$ ( as shown in fig. 2c) Measured $\mathrm{S}_{11}$ in $\mathrm{dB}$ wrt to frequency in $\mathrm{GHz}$ (as shown in fig. 4).

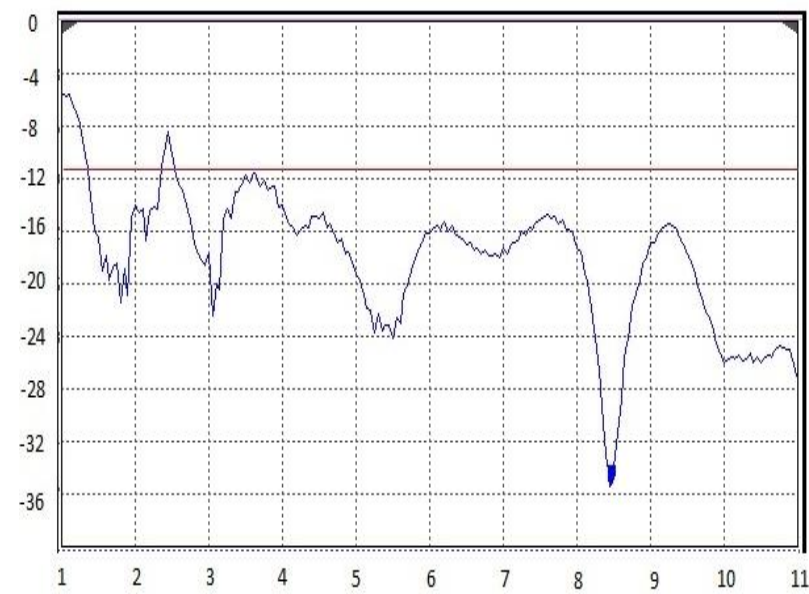

Fig 4: $S_{11}$ The measured and simulated results

The simulated radiation efficiency and maximum gain is shown in fig. 5 . The gain found to be around $4 \mathrm{dBi}$, between 2 to $7 \mathrm{dBi}$, for the entire required bandwidth and for the same frequency range radiation efficiency varies between 80 to $95 \%$, So this antenna is efficient enough for the entire band of frequency from $1.4 \mathrm{GHz}$ to $11 \mathrm{GHz}$.

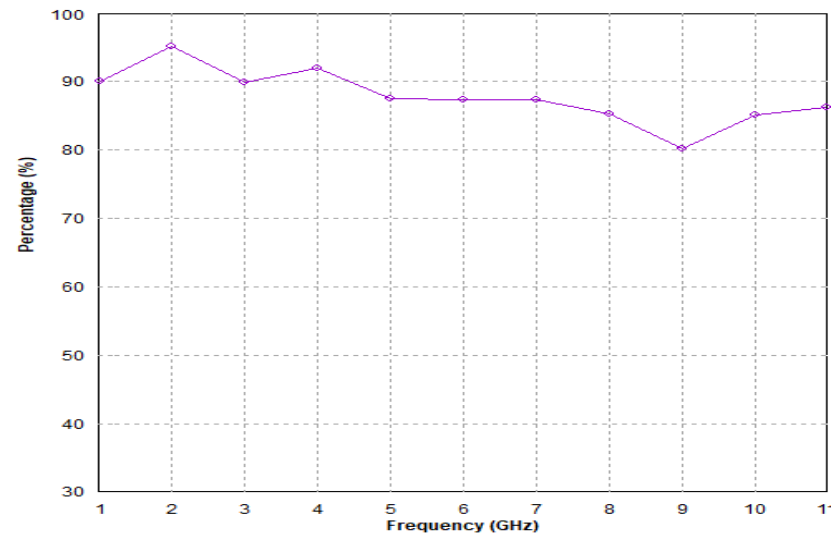

Radiation Efficiency

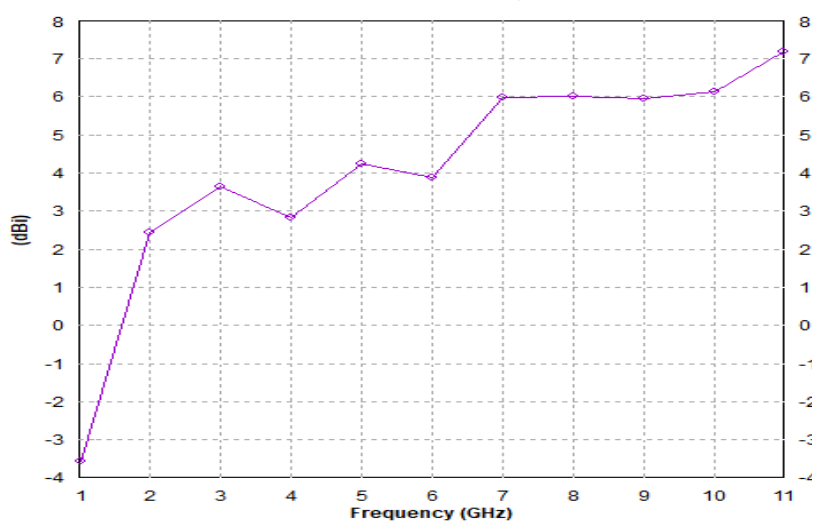

Maximum gain

Fig5: simulated radiation efficiency and maximum gain
The simulated 3D-radiation pattern is observed for entire bandwidth and the experimental result had been observed over one frequency at $3 \mathrm{GHz}$. The radiation patterns are shown in fig. 6 .

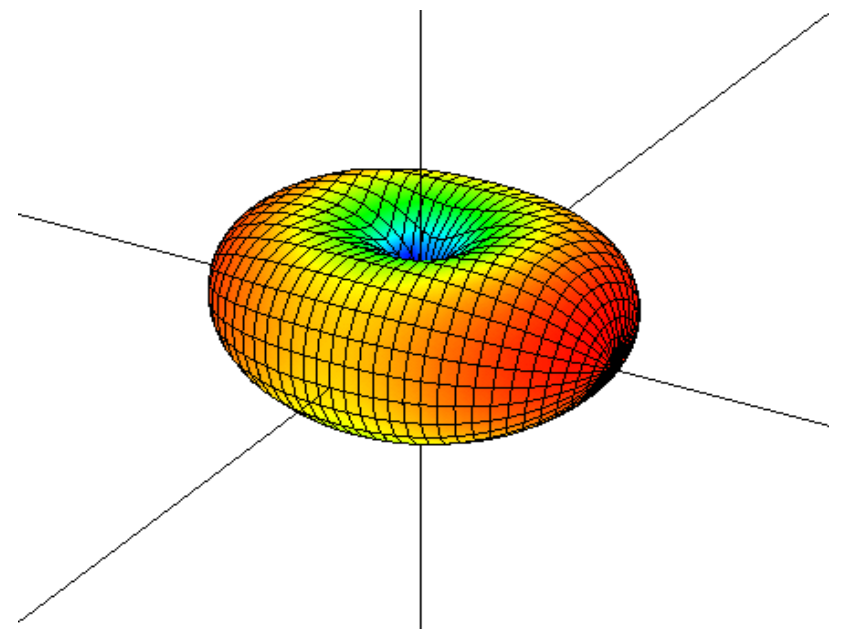

(a). 3D simulated radiation pattern at $3 \mathbf{G H z}$

The presented antenna shows radiation pattern as omni-directional on azimuthal plane and the radiation pattern is 'Figure of Eight' on elevation plane. The measured and simulated radiated field patterns are in good agreement.

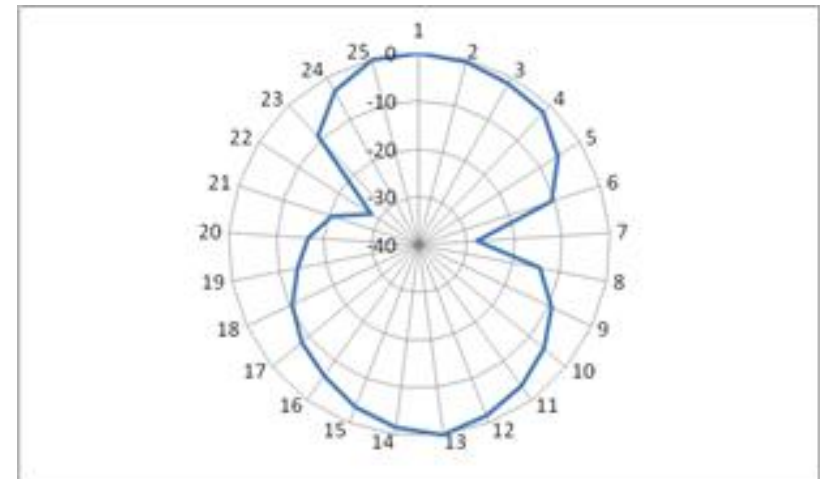

On elevation plane

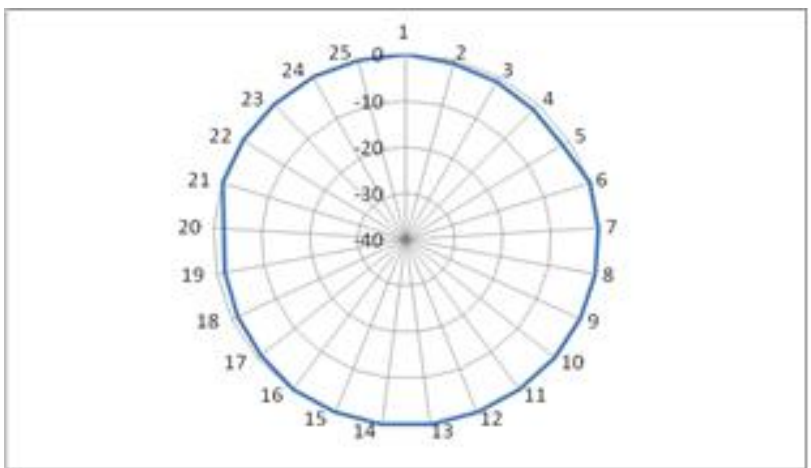

On azimuthal plane

(b). Measured radiation pattern at $3 \mathrm{GHz}$ Fig 6: Radiation pattern at $3 \mathrm{GHz}$

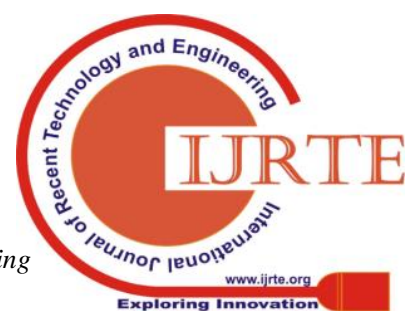




\section{CONCLUSION}

To improve the bandwidth of antenna a simple way is to use multi-resonators with overlapping of multi-resonance with better impedance matching. The proposed antenna has been designed, optimized with help of simulator then fabricated the prototype. The simulated results were validated in the laboratory. The designed antenna is showing good radiation characteristics over the entire UWB and beyond. This is very handy band for many wireless systems including the UWB.

\section{REFERENCES}

1. KP Ray, SS Thakur, Printed annular ring with circular patch monopole UWB antenna ,2012 International Conference on Advances in Computing and Communications , pp. 270-273, IEEE, 2012/8/9

2. KP Ray, SS Thakur, RA Deshmukh, Broadbanding a printed rectangular monopole antenna 2009 Applied Electromagnetics Conference (AEMC), pp.1-4, 2008

3. Sanjay Singh Thakur5, ShraddhaKarnik, HimanshiBansod, ShubhamBajirao, Vivek Philip, Design of Multiband Antenna, JASC: Journal of Applied Science and Computations, Volume 5, Issue 10, pp799-801, October2018

4. Kamala Prasan Ray, Design aspects of printed monopole antennas for ultra-wideband applications, , International Journal of Antennas and Propagation, Volume, 2008, Publisher-Hindawi, 2008.

5. Rakhesh Singh Kshetrimayum, Printed Monopole Antennas for Multiband Applications, International Journal of Microwave And Optical Technology Vol. 3, No. 4, pp. 474-480, SEPTEMBER 2008.

6. Sani Mubarak Ellis, Abdul-Rahman Ahmed, Kponyo Jerry, J. Nourinia, ChangizGhobadi, B. Mohammadi, Miniaturized printed monopole antenna with a linked ground plane and radiator, Electronics Letters 54(11), April 2018

7. NeelaveniAmmalMurugan,

RamachandranBalasubramanian , and HanumanthaRaoPatnam, Printed Planar Monopole Antenna Design for Ultra-Wideband Communications, Radioelectronics and Communications Systems, 2018, Vol. 61, No. 6, pp. 267-273, 2018.

8. G Kumar, KP Ray, Broadband micro strip antennas, Artech house, 2003

9. Zealand software Inc., IE3D, 2016 